\title{
High-Fat Diet Induced Anxiety and Anhedonia: Impact on Brain Homeostasis and Inflammation
}

\author{
Sophie Dutheil', Kristie T Ota', Eric S Wohleb', Kurt Rasmussen² and Ronald S Duman ${ }^{*, 1}$ \\ 'Department of Psychiatry, Yale University School of Medicine, New Haven, CT, USA; 'Eli Lilly and Company, Lilly Corporate Center, Indianapolis, IN, USA
}

\begin{abstract}
Depression and type 2 diabetes (T2D) are highly comorbid disorders that carry a large public health burden. However, there is a clear lack of knowledge of the neural pathological pathways underlying these illnesses. The present study aims to elucidate the molecular mechanisms by which a diet rich in fat can cause multiple complications in the brain, thereby affecting intracellular signaling and gene expression that underlie anxiety and depressive behaviors. The results show that a high-fat diet (HFD; 16 weeks) causes anxiety and anhedonic behaviors. Importantly, the results also show that 4 months of HFD causes disruption of intracellular cascades involved in synaptic plasticity and insulin signaling/glucose homeostasis (ie, Akt, extracellular signal-regulated kinase (ERK), P70S6K), as well as increased corticosterone levels and activation of the innate immune system, including elevation of inflammatory cytokines (ie, IL-6, IL-I $\beta$, TNF $\alpha$ ). Interestingly, the rapid acting antidepressant ketamine reverses the behavioral deficits caused by HFD and activates ERK and P70S6 kinase signaling in the prefrontal cortex. In addition, we found that pharmacological blockade of the innate immune inflammasome system by repeated administration of an inhibitor of the purinergic P2X7 receptor blocks the anxiety caused by HFD. Together these studies further elucidate the signaling pathways that underlie chronic HFD exposure on anxiety and depressive behaviors, and identify novel therapeutic targets for patients with metabolic disorder or T2D who suffer from anxiety and depression.

Neuropsychopharmacology (2016) 4I, I874-1887; doi:10.1038/npp.20 15.357; published online 13 January 2016
\end{abstract}

\section{INTRODUCTION}

Processed foods rich in saturated fat have become a major part of our diet and culture in Western countries, contributing to an obesity epidemic associated with a multitude of health complications and excessive economic costs. People with an unhealthy high-fat diet (HFD) lifestyle are more likely to develop obesity, chronic systemic illnesses like cardiovascular disease, type 2 diabetes (T2D), and hypertension (Anderson et al, 2001). In the long term, consuming excessive amounts of foods with saturated fat affects the homeostasis of the whole body, including brain health. Cumulative evidence shows that a diet rich in fatty foods decreases hippocampus volume, impairs cognitive function including memory, psychomotor efficiency, attention in humans, and, more importantly, increases the vulnerability to depression and anxiety (Anderson et al, 2001; Jacka et al, 2015).

To date, overlap in the pathophysiology of T2D and depression has been reported in neuropsychological and neuroimaging studies (den Heijer et al, 2003; Kamiyama et al, 2010; Last et al, 2007), and is supported by findings in

* Correspondence: Dr RS Duman, Department of Psychiatry, Yale University School of Medicine, 34 Park Street Room S308, New Haven, CT 06519, USA, Tel: +203974 7723, Fax: +203974 7724, E-mail: ronald.duman@yale.edu

Received I October 2015; revised 12 November 2015; accepted 28 November 2015; accepted article preview online 14 December 2015 rodent models that allow better characterization of the fine pathological consequences induced by HFD. More precisely, rats or mice exposed to chronic HFD develop successive levels of impairments, including inflammation, high corticosterone (CORT) levels, and deficits comparable to accelerated brain aging in limbic structures where insulin and glucocorticoïd receptors are particularly abundant (De Souza et al, 2005; Machida et al, 2012; Stienstra et al, 2011). Interestingly, some of these HFD-induced alterations are also described in stress-induced models of depression that are relevant to findings in humans, including increased CORT, low-grade inflammation, neuronal atrophy, decreased hippocampal neurogenesis, and decreased neurotrophic factor expression (Duman et al, 2012; Ghasemi et al, 2012; Salim et al, 2012). Clinical studies have reported that T2D patients are at higher risk for depressive and conversely that depressed patients are at high risk for T2D (Anderson et al, 2001). Rates of mortality are also 1.5 -fold higher for patients with T2D and depression (van Dooren et al, 2013). However, despite the critical impact on public health, the exact molecular and cellular signaling alterations increasing the susceptibility to develop both diseases remain incompletely defined, and new biomarkers relevant to both diagnosis and treatment response are needed.

We recently reported that disruption of the mechanistic target of rapamycin complex 1 (mTORC1) pathways contributes to the synaptic and behavioral deficits caused by chronic stress ( $\mathrm{Li}$ et al, 2011; Ota et al, 2014a). The importance of this pathway is further demonstrated by 
evidence that the rapid acting antidepressant ketamine stimulates mTORC1 signaling and that this pathway is required for the behavioral actions of ketamine ( $\mathrm{Li}$ et al, 2010, 2011). Based on this evidence, we examined the influence of HFD on mTORC1 signaling in hippocampus and prefrontal cortex (PFC), two key structures involved in both depressive disorder and brain insulin resistance, and the ability of ketamine to reverse the behavioral deficits caused by HFD.

Further, because T2D and metabolic disorders are known to cause low-grade inflammation in peripheral systems as well as the central nervous system, and likewise, levels of inflammatory cytokines are increased in the blood of depressed patients, and in both blood and brain in rodents exposed to chronic stress models of depression (Iwata et al, 2013; Koo et al, 2010; Schmidt et al, 2011), we investigated the influence of HFD on the innate immune-inflammasome system and the effect of a pharmacological inhibitor of this system, a purinergic P2X7 receptor antagonist, to reverse the behavioral deficits caused by HFD.

Our results offer novel insights about potential treatments and prevention strategies for patients with T2D, revealing that HFD-induced anxiety and depressive behavior can be reversed by treatments targeting the mTORC1 signaling pathway and the innate immune response.

\section{MATERIALS AND METHODS}

\section{Animals and Diets}

Male Sprague-Dawley rats weighing 200-230g at the beginning of the HFD administration were housed in pairs in rectangular polypropylene cages with standard laboratory bedding and conditions with a 12-h light/dark cycle and ad libitum access to food and water. Animal use and procedures were in accordance with the National Institutes of Health guidelines and approved by the Yale University Animal Care and Use Committees. During 1 week acclimation period, animals were first fed with a standard chow diet providing $4.10 \mathrm{kcal} / \mathrm{g}$ and then divided into two homogenous groups with balanced weight. Based on studies reporting that $45-60 \%$ of HFD causes metabolic alterations that are clinically relevant to T2D, we chose $60 \%$ HFD (20\% CHO, $60 \%$ fat, D12492, Research Diets) that provided $5.24 \mathrm{kcal} / \mathrm{g}$ of diet (HFD group, $n=64$ ). For the control diet (CD), we chose a low-fat purified diet containing the same ingredients matched with the composition of the high-fat formula, including sucrose and providing $3.85 \mathrm{kcal} / \mathrm{g}$ of diet, of which $10 \%$ from fat (D12450J, Research Diets, CD group, $n=64$ ). This CD avoids problems encountered when normal laboratory chow (cereal based diet) is used as a low-fat 'control' diet, which does not account for other components such as phytoestrogens from soy that can influence anxietyrelated behaviors, water and food intake, locomotor activity (LA), learning and memory, and blood insulin (Lephart et al, 2004; Warden and Fisler, 2008).

Two time points after the beginning of the special diets were selected based on the literature (De Souza et al, 2005; Pratchayasakul et al, 2011): an intermediate time (8 weeks) when peripheral insulin resistance has developed, and a long-term time point (16 weeks) when a diabetic-like state has developed associated with peripheral and brain insulin resistance (16 weeks). Body weight was measured once a week. During the last 3 weeks, animals were single-housed for behavioral testing.

\section{Study Design and Drug Administration}

The first experiment was conducted to study the baseline effects of HFD; $16 \mathrm{CD}$ and $16 \mathrm{HFD}$ rats were used for western blot analysis after 8 or 16 weeks; another $16 \mathrm{CD}$ and 16 HFD rats were used for molecular, biochemical, and behavior studies and killed after 16 weeks; and $8 \mathrm{CD}$ and 8 HFD rats were used for glucose tolerance tests. For behavioral studies, novelty suppressed feeding test (NSFT) was the first test performed and sucrose preference test (SPT) the last preceding sacrifice.

In a second experiment, $16 \mathrm{CD}$ and $16 \mathrm{HFD}$ rats received either ketamine $(20 \mathrm{mg} / \mathrm{kg})$ or vehicle (saline; i.p.), once at the end of 15 weeks and were subjected to behavioral testing over the next 6 days. The behavioral tests were performed in the following order: d0, first ketamine injection at $20 \mathrm{mg} / \mathrm{kg}$ or saline; d1, food deprivation; d2, NSFT and home cage feeding (HCF); d3, locomotor activity (LMA); d4, female urine sniffing test (FUST); d5, novel object recognition (NOR) training with test $1 \mathrm{~h}$ later. Rats received an addition dose of ketamine every 6 days until the behavioral testing was completed. This treatment schedule is based on the clinical studies demonstrating that a single dose of ketamine produces significant antidepressant effects for $\sim 1$ week and then most patients relapse (Berman et al, 2000; Romeo et al, 2015; Zarate et al, 2006). Hence, the order of the additional injections and tests was: d6, second ketamine or saline injection; d7, elevated plus maze (EPM); d12, third ketamine or saline injection; d13, sucrose $1 \%$ habituation until d17; d17-18, sucrose over water choice training, followed by the fourth ketamine or saline injection; d19, SPT; d21, open field test (OFT) for $15 \mathrm{~min}$; d23, fifth ketamine or saline injection; $\mathrm{d} 24$, staggered sacrifice $24 \mathrm{~h}$ after last injection.

In a third experiment, $16 \mathrm{CD}$ and $16 \mathrm{HFD}$ rats received either P2X7 antagonist A804598 (5 mg/kg, Eli Lilly, Indiana, IN) or vehicle (10\% dimethyl sulfoxide, $90 \%$ Polyethylene glycol 400; i.p.), twice a day during 25 days, starting from week 12 to 16 after the start of the CD or HFD. Behavioral testing started 16 days after the beginning of the injections and lasted 8 days with tests performed in the following order: $\mathrm{d} 0$, first injection; $\mathrm{d} 15$, food deprivation; d16, NSFT and HCF; d18, OFT; d19, EPM; d25, staggered sacrifice 4 h after last morning injection.

\section{Glucose Tolerance Test (GTT)}

GTT were conducted at different time points $(1,4,8,12$, and 16 weeks of HFD or CD) on a separate cohort of animals. After an overnight fast, rats were anesthetized with pharmaceutical grade pentobarbital (Nembutal, $50 \mathrm{mg} / \mathrm{kg}$, i.p.). Then, a drop of blood was collected from a tail vein and expressed on a blood glucose test strip (Onetouch, Verio) to obtain a baseline value using a Glucometer (Onetouch Verio IQ) followed by injection of $25 \%$ glucose $(2 \mathrm{~g} / \mathrm{kg}$ body weight, i.p.), and blood glucose levels were measured at 10, $20,30,60,90$, and $120 \mathrm{~min}$ after glucose injection. 


\section{Behavioral Tests}

The following behavioral tests were conducted at the time points indicated in the text and figures. All scoring was conducted blind to the treatment group.

NSFT and HCF. The NSFT was performed as previously described (Warner-Schmidt and Duman, 2007). Rats were food-deprived overnight and placed in an open field $\left(76.5 \times 76.5 \times 40 \mathrm{~cm}^{3}\right)$ with a small amount of food pellets that included both the CD and HFD in the center to avoid any olfactory bias. At the time of the test, animals were exposed to the open field for the first time (novelty) and allowed to explore for $15 \mathrm{~min}$. The latency for the animal to approach and take its first bite of the food was recorded. Home cage food intake was also measured as a control over a period of $10 \mathrm{~min}$ following the end of the entire test session.

Elevated plus maze. Activity in an EPM apparatus with two open and two closed arms was measured over a total of 10-min period using the AnyMaze tracking system.

Open field test. Activity in an open field was measured over a 8-min period with AnyMaze Software in open field boxes $\left(76.5 \times 76.5 \times 40 \mathrm{~cm}^{3}\right.$ height $)$. Time spent in the margin and center, total distance, and mean speed were measured.

Sucrose preference test. Rats were exposed to a palatable sucrose solution (1\%; Sigma) for $96 \mathrm{~h}$. The day of the test, rats were placed in a quiet room where cages were in a dimly lit area. After $4 \mathrm{~h}$ of water deprivation, rats were exposed to two identical bottles, one filled with $1 \%$ sucrose and the other with plain water. Sucrose and water consumption were determined by measuring the change in the volume of fluid consumed. Sucrose preference was defined as the ratio of the volume of sucrose versus total volume of sucrose plus water consumed during the $1 \mathrm{~h}$ test.

Female urine sniffing test. This test was conducted according to the published procedures (Malkesman et al, 2010). Rats were brought to a well-ventilated testing room and $30 \mathrm{~min}$ later were habituated for $60 \mathrm{~min}$ to a sterile cotton-tipped applicator placed in their home cage. For the test, rats were first exposed to a new cotton tip dipped in sterile water; $45 \mathrm{~min}$ later, rats were then exposed to another cotton tip infused with fresh urine collected from females of the same strain. Prior studies have reported that males have no preference for receptive $v s$ non-receptive female odors, and that females in all states of estrus elicit higher investigation from males than ovariectomized females (Macbeth et al, 2009), so the estrous cycle was not monitored. Male behavior was video recorded and latency to first sniff the cotton tip and total time spent sniffing the cotton-tipped applicator were determined. Time spent biting the cotton-tip was excluded from the recording time.

Novel object recognition. Rats were habituated to the arena $\left(76.5 \times 76.5 \times 40 \mathrm{~cm}^{3}\right)$ in the absence of objects for $10 \mathrm{~min}$ for two consecutive days. The following day, rats were placed in the arena in the presence of two identical objects placed $13 \mathrm{~cm}$ from the walls of the open field, and were allowed to explore for $15 \mathrm{~min}$. One hour later, they were placed back in the apparatus with one of the previously explored objects (familiar), and one novel object. Rats were allowed to explore for $8 \mathrm{~min}$. Positioning (left side versus right side) of the novel object was balanced between groups. Behavior was video recorded and exploratory behavior was determined. Exploration was defined as a rat having its nose close to the object. Climbing or sitting on objects was not scored as object exploration.

Locomotor activity. LMA was measured for $30 \mathrm{~min}$ in an empty rat cage equipped with automated activity meters (Digiscan animal activity monitor; Omnitech Electronics, Columbus, Ohio) as previously described (Warner-Schmidt and Duman, 2007).

\section{CORT ELISA}

Trunk blood was collected into $12 \mathrm{ml}$ Falcon tubes and then allowed to sit at room temperature for $1 \mathrm{~h}$ before being placed on ice. Samples were centrifuged at $1500 \mathrm{~g}$ for $10 \mathrm{~min}$. The supernatant was collected and stored at $-80^{\circ} \mathrm{C}$ until use. Levels of CORT (ng/ml) were determined using an Enzo Life Sciences ELISA kit according to the manufacturer's instruction and a $\mu$ Quant microplate reader (BioTek Instrument Inc, Winooski, VT). Samples were run in duplicates.

\section{Cytokine Assay}

Protein levels of hippocampal IL-1 $\beta$, IL-6 and TNF $\alpha$ were measured using a V-Plex Meso Scale Discovery Multiplex spot assay and analyzed with MSD Discovery Workbench software (Meso Scale Discovery, Gaithersburg, MD, USA). All samples were run in duplicates or triplicates.

\section{Quantitative Real-Time PCR}

RNA from half hippocampus or half PFC was purified using RNAqueous (Ambion), and 500 ng of total RNA was used for cDNA synthesis (Genisphere). The purity and concentration of DNA was measured with a Nanodrop spectrophotometer; the OD260/280 was limited within 1.8-2.0. Genespecific primers were designed using Primer 3 software for ADAM17, CYP11B1, Insulin 2, Insulin receptor, IL-6, IL-1 $\beta$, TNF $\alpha$, Toll-like receptor (TLR) 2, and TLR4 (see primer sequence in Table 1). PCR was run on the ABI 7900HT (Applied Biosystems) using SYBR Green (Qiagen) on 384-well plates from applied biosystems (Life Technologies). $\mathrm{Ct}$ values of genes of interest were normalized to that of housekeeping gene (HMBS).

\section{Western Blotting}

Sections of PFC from one half of the brain were dissected and immediately homogenized in a solution containing $0.32 \mathrm{M}$ sucrose, $20 \mathrm{mM}$ HEPES ( $\mathrm{pH} 7.4$ ), $1 \mathrm{mM}$ EDTA, $1 \times$ protease inhibitor cocktail, $5 \mathrm{mM} \mathrm{NaF}$, and $1 \mathrm{mM}$ sodium vanadate. Synaptoneurosome-enriched fractions were prepared and sonicated in lysis buffer. The same procedure was performed for sections of hippocampus from one half of the brain. Protein concentration was determined by BCA protein assay (Pierce Biotechnology). Equal amounts of protein were 
Table I Primers Designed for Real-time qPCR

\begin{tabular}{|c|c|c|}
\hline $\begin{array}{l}\text { Gene of interest } \\
A D A M / 7\end{array}$ & F-GTTCGCATTAAGCCGTTCC & R-TGGGGTCTTCCTGGATGGTG \\
\hline$I L-\mid \beta$ & F-GTGTCACTCATTGTGGCTGTGG & R—GGGAACATCACACACTAGCAGGTC \\
\hline IL-6 & F-AGAAAAGAGTTGTGCAATGGCA & R-GGCAAATTTCCTGGTTATATCC \\
\hline$T N F \alpha$ & F-CAAGGAGGAGAAGTTCCCA & R-TTGGTGGTITGCTACGACG \\
\hline TLR4 & F-CCAGAATGAGGACTGGGTGAGAAA & R-CCACCACAATAACTTTCCGGCTCTTG \\
\hline INSR & F-GCTTCTGCCAAGACCTTCAC & R-TAGGACAGGGTCCCAGACAC \\
\hline INS2 & F-CAGCACCTTTGTGGTTCTCA & R-CAGTGCCAAGGTCTGAAGGT \\
\hline NLRP3 & F-CAGGGCTAAAGCAGCAGATGG & R-TCCGCAGCCAATGAACAGAG \\
\hline HMBS & F-GGAGGTCCGAGCCAAGGACCAG & R-CTGGCACGCTACAGCCTCCTTCC \\
\hline
\end{tabular}

Abbreviations: IL- I $\beta$, interleukin- I $\beta$; IL-6, interleukin 6; TLR2, Toll-like receptor 2; TLR4, Toll-like receptor 4; TNF $\alpha$, tumor necrosis factor $\alpha$.

Table 2 Western Blot Antibodies References and Concentrations

\begin{tabular}{llcl}
\hline Primary antibody & Source & Reference & Dilution \\
\hline p-Akt308 & Cell Signaling & 4056 & I:1000 \\
Total Akt & Cell Signaling & $927 \mid$ & I:1000 \\
P-ERK & Cell Signaling & 4370 & I:1000 \\
Total ERK & Cell Signaling & 4695 & I:1000 \\
p-mTOR & Cell Signaling & 5536 & I:1000 \\
Total mTOR & Cell Signaling & 2972 & I:1000 \\
p-P70S6K & Cell Signaling & 9205 & I:1000 \\
Total P70S6K & Cell Signaling & 2708 & I:1000 \\
\hline
\end{tabular}

loaded and separated on a $7.5 \%$ SDS-PAGE gel. After electrophoresis, the proteins were electrically transferred to nitrocellulose membranes and blots were incubated in the appropriate primary antibody for phosphorylated (phospho)-Akt, phospho-extracellular signal-regulated kinase (ERK), phospho-mTOR, phospho-P70S6K, and their respective total protein levels (see references and dilutions in Table 2) and developed using enhanced chemiluminescence (GE Biosciences). The intensity of the protein bands was quantified using image analysis software (ImageJ 1.35, National Institute of Mental Health). For each blot, the background signal was determined by tracing an unlabeled area adjacent to each band with the same square and subtracting this value from the target band. Resultant values were normalized to the average signal for the total (nonphosphorylated) protein levels to simplify gel analysis and reduce inter and intra-gel variability. Data are expressed as fold change $v s$ control levels.

\section{Data Analysis}

Data were analyzed using SPSS Software (SPSS). Results are presented as mean $\pm S E M$ and were analyzed using two-tailed Student's $t$-tests for two-group comparisons (CD vs HFD), or two-way ANOVA with diet (CD vs HFD) and treatment (saline or vehicle vs $\mathrm{P} 2 \mathrm{X} 7$ antagonist or ketamine) followed by a post hoc pair wise multiple comparison procedure using the Fischer's least significant difference (LSD) method if the interaction was significant. The homogeneity of variance was determined for each data set using GraphPad Prism (GraphPad Software, La Jolla, California, USA). Because measured serum corticosterone levels did not follow a normal distribution, values were subjected to logarithmic transformation. Log-transformed data were then used for further $t$-test analysis. Differences were considered significant if $P<0.05$. Outliers were removed using the median absolute deviation equation (median plus or minus 2.5 times the MAD method for outlier detection), a more robust measure of dispersion than the mean \pm 2 or 3 standard deviation (Leys et al, 2013).

\section{RESULTS}

\section{HFD Exposure Increases Bodyweight and Leads to Glucose Intolerance}

Before diet exposure, the groups were balanced and average weights were similar (CD: $168.07 \mathrm{~g}, \mathrm{HFD}: 169.07 \mathrm{~g}$ ). After 1 day of exposure to the new diet, rats fed HFD consumed significantly more $\mathrm{kcal}(+56.57 \% \mathrm{kcal})$ than the CD group (CD: $80.08 \mathrm{kcal}, \mathrm{HFD}: 125.39 \mathrm{kcal}, P=0.0019)$, suggesting that HFD is more palatable than the CD (Figure 1a). Three weeks later, HFD animals had decreased their average daily food intake so that caloric intake levels were comparable to the CD group. This diminished caloric intake was observed until sacrifice (Figure 1a).

Nine weeks of HFD were necessary to cause a significant weight gain between the two groups, with the HFD rats $+7.45 \%$ compared with controls (CD: $561.1 \mathrm{~g}$, HFD: $602.93 \mathrm{~g}$, $P=0.0323$ ); the HFD group weight then remained higher throughout the experiment (Figure 1b). GTTs, a diagnostic tool for diabetes and indicator of metabolic efficiency and insulin resistance, were performed $1,4,8,12$, and 16 weeks of HFD. Glucose administration initially increased glucose blood levels in both $\mathrm{CD}$ and HFD rats; a reduction was observed in the CD group 60 min after injection and then 

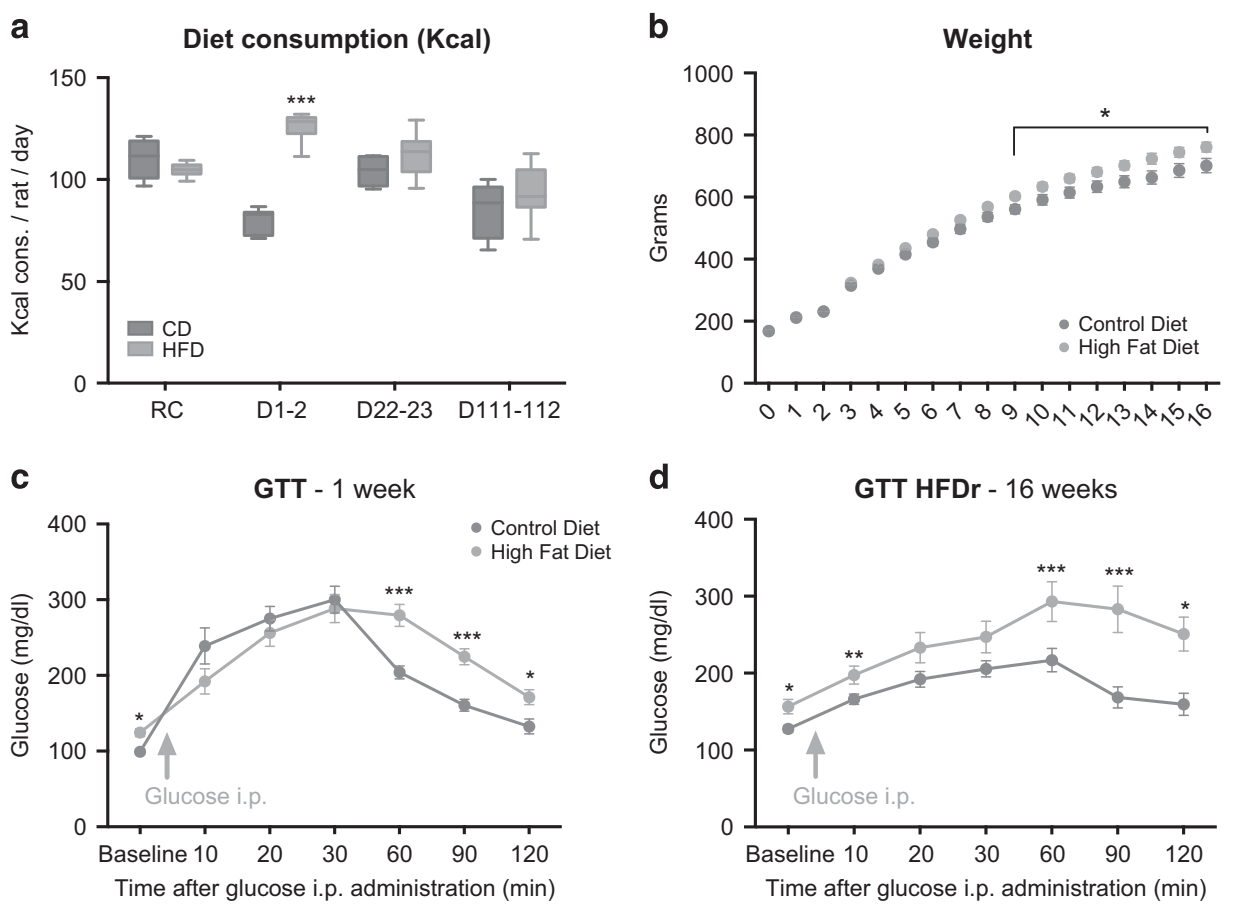

Figure I HFD exposure increases bodyweight, glucose tolerance, and hyperglycemia. (a) Diet consumption (kcal) by rat and per day on regular chow (RC) before special diet exposure, during the first $24 \mathrm{~h}$ (days I-2) of control diet (CD) or high-fat diet (HFD), 22 days later (D22-23) and after I6 weeks (DI I |-| | 2). (b) Bodyweight from day 0 to the I6th week of a cohort of rats fed CD or HFD. (c) Glucose tolerance test (GTT) results after I week of special diet consumption. (d) GTT results after 16 weeks of special diet consumption in control and HFD-responsive (HFDr) groups. $* P<0.05$, **** $<0.00$ I when compared with the corresponding CD group. Results are presented as the mean \pm SEM.

returned to baseline by $120 \mathrm{~min}$. HFD rats displayed a significant difference from CD after 1 week (ie, reduced glucose removal), with a greater effect after 16 weeks of HFD (Figure 1c).

After 1 week of HFD, animals had a higher baseline fasting glucose level than controls (Figure 1c), possibly due to overconsumption of the more palatable HFD, but during the intermediate times $(4,8$, and 12 weeks) baseline levels were comparable with controls (not shown). After 16 weeks, there was a trend toward increased glucose levels in the HFD group compared with CD (not shown). At that time point, rat weight was positively correlated with the morning glucose level (Pearson correlation: $\mathrm{r}_{(14)}=0.551 ; P=0.0363$ ), suggesting that obesity and hyperglycemia could be related. Thus, we decided to look at HFD-responsive rats and to exclude from this analysis rats that had weights lower than the heaviest controls ( $<750 \mathrm{~g}$ ); there were two such rats, referred to as HFD-resistant that were excluded from analysis in Figure 1d: $n=2)$. Results showed significant higher morning glucose values in HFD-responsive rats compared with controls (CD: 127.375, HFDr, $154.6 \mathrm{mg} / \mathrm{dl}, \quad P=0.0259$, Figure 1d), suggesting that rats that become obese under HFD develop diet-induced hyperglycemia.

\section{HFD Consumption Increases Anxiety and Anhedonic Behaviors}

To determine whether our HFD contributes to behavioral changes, we first analyzed anxiety and anhedonic behaviors with several commonly used tests, including NSFT, EPM, OFT, and SPT. After 8 weeks of HFD, there were no significant effects on these behaviors (non-shown). However, after 15-17 weeks, we found that HFD consumption significantly altered behavior in several models of anxiety without effecting overall ambulatory activity, as assessed in either an empty cage for $30 \mathrm{~min}$ (Figure $2 \mathrm{~b}$ ) or in an open field for $8 \mathrm{~min}$ (not shown). HFD increased latency to feed in the NSFT (CD: $309.4 \mathrm{~s}$, HFD rats: $496.8 \mathrm{~s}, P=0.0002$, Figure 2c), but had no effect on HCF, a control for the NSFT (Figure 2d). HFD also reduced the number of entries in the center of an open field (CD: 12.8 HFD rats: 8.07, $P=0.0372$, Figure 2e), which can be used as a readout for anxiety-like behavior. In the EPM, there was a nonsignificant trend toward decreased time spent in open arms of $\sim 20 \%$ in HFD rats (Figure $2 \mathrm{f}$ ). We also examined the influence of HFD on NOR, an object memory test that takes advantage of a rat's preference to explore novel objects. When tested with a novel object $1 \mathrm{~h}$ after training, HFD rats spent less time exploring the novel object over the familiar (CD: 58.59\%, HFD: $38.86 \%, P=0.0014$, Figure $2 \mathrm{j}$ ).

We then assessed preference for a sweetened solution in the SPT, a measure of anhedonic behavior that is a core symptom of depression. Whereas $C D$ rats displayed a preference for sucrose solution, HFD rats showed no preference over plain water (CD: $87.01 \%$ sucrose preference, HFD rats: $61.08 \%, P=0.0195$, Figure $2 \mathrm{~g}$ ). This indicates that HFD animals lost their natural preference for a sweetened solution, suggesting a loss of interest in pleasurable experiences. To confirm this result, we used another test for anhedonia, the FUST, which is based on the attraction of male rodents to pleasurable pheromones/odors in female urine and serves as a measure of reward-seeking behavior in 


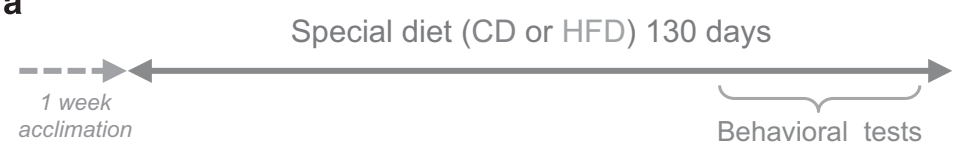

b

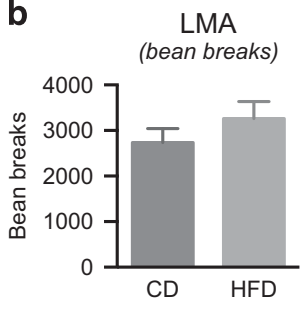

c

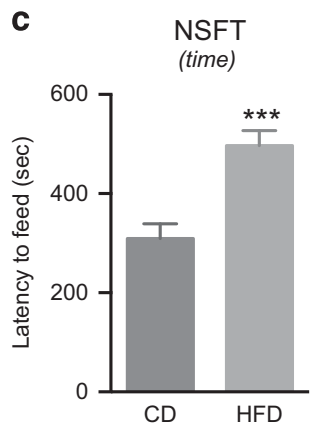

d

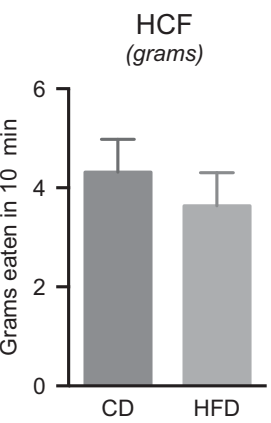

e
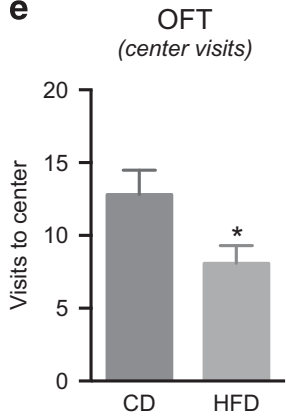

h
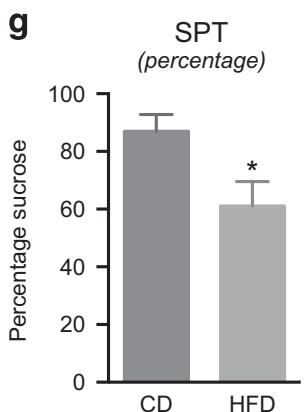

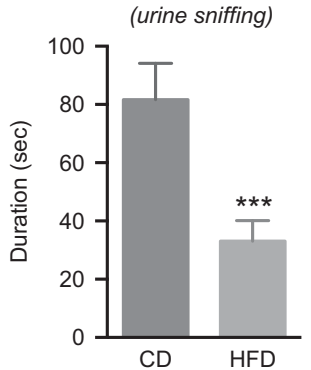

i

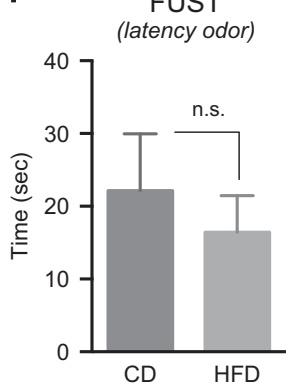

f

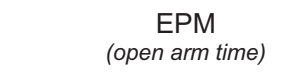

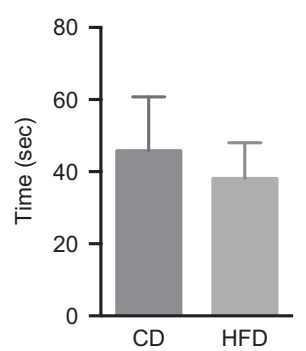

j
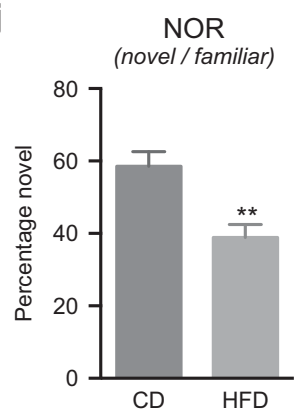

Figure 2 HFD increases anxiety and anhedonia behaviors. (a) Experimental paradigm of CD or HFD given for 16 weeks while behavioral testing started at week 14. Influence of HFD on (b) locomotor activity (LMA), anxiety assessed in the (c) novelty suppressed feeding test (NSFT), and its control (d) home cage feeding (HCF); (e) open field test (OFT) and ( $f$ ) elevated plus maze (EPM). HFD exposure also leads to anhedonic behaviors as assessed with (g) sucrose preference test (SPT), (h) female urine sniffing test (FUST), and (i) its control for olfactory sensitivity. (j) Influence of HFD on novelty object recognition (NOR). Results are presented as the mean \pm SEM, $n=16$ per group. $* P<0.05$, $* * P<0.0$ l, $* * * P<0.001$ compared with the CD (two-tailed Student's $t$-tests).

rodents (Malkesman et al, 2010). We found that HFD rats spent less time sniffing female urine than $C D$ rats (CD: $81.65 \mathrm{~s}, \mathrm{HFD}: 33.08 \mathrm{~s}, P=0.0022$, Figure $2 \mathrm{~h}$ ); there was no difference in the latency to sniffing (CD: $22.15 \mathrm{~s}$, HFD: $16.43 \mathrm{~s}$, Figure 2i). These results indicate that HFD rats show symptoms that mimic those of human sufferers of depression, such as failing to take pleasure in activities that are normally enjoyable.

\section{HFD Disrupts Expression of Genes and Proteins Involved in Insulin Signaling, mTORC1/Synaptic Plasticity, and Corticosterone Synthesis}

To elucidate the signaling mechanisms underlying the effects of HFD, we measured the expression of proteins and genes involved in synaptic plasticity, glucose metabolism, and corticosterone synthesis (Figure 3a). First, we found an increase in mRNA levels of the insulin receptor and a trend for increased insulin 2 in the hippocampus in after 16 weeks of $\operatorname{HFD}(P=0.022$ and $P=0.08$, Figure $3 g$ and $h)$. This could represent a compensation for development of insulin resistance.
Insulin receptor activation leads to phosphorylation and stimulation of several kinases, including Akt and ERK that stimulate mTORC1 signaling. Phosphatidylinositol (PI) 3-kinase/Akt is also one of the main insulin signaling pathways and has a role in insulin stimulation of glucose transport. Levels of each phosphorylated proteins were standardized to total levels of the same protein. We found that HFD exposure for 8 weeks significantly decreased levels of phospho-Akt in a synaptoneurosome-enriched preparation from hippocampus $(P=0.0181)$; there was a nonsignificant reduction of phospho-Akt at week 16 (Figure $3 \mathrm{c}$ ). The results also show that HFD for 8 weeks increases levels of phospho-ERK (including ERK1 and ERK2) by $36.8 \%(P=0.018)$, with a return to $\mathrm{CD}$ levels by week 16 (Figure 3d). Western blot analysis of phospho-mTOR expression did not show any effect of HFD (Figure 3e), but downstream mTORC1 enables the translation of synaptic proteins by activating P70S6 kinase. This pathway has a key role in synaptic plasticity and is involved in the pathophysiology and treatment of stress-induced behaviors (Li et al, 2011; Ota et al, 2014a). Here, the results indicate that phospho-P70S6K levels are first increased approximately twofold after 8 weeks $(P=0.0109)$, but then significantly 
a
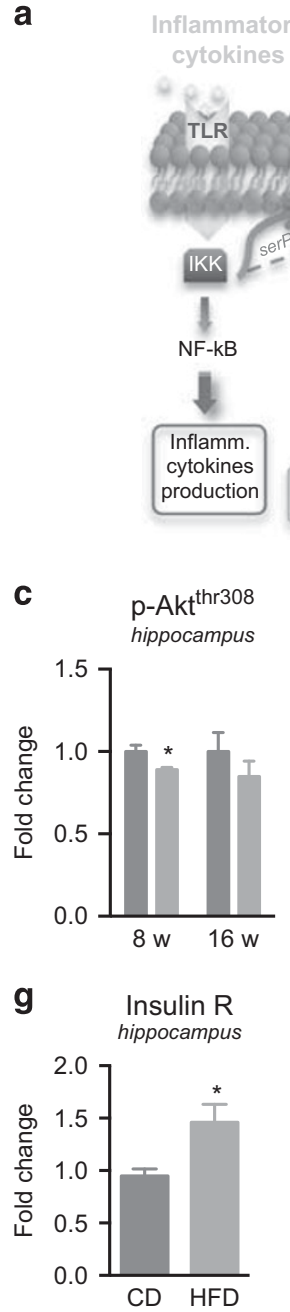

d

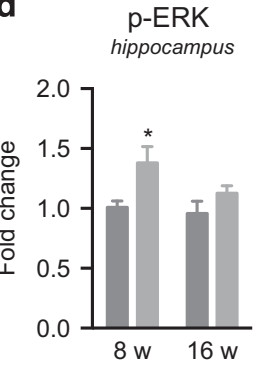

h

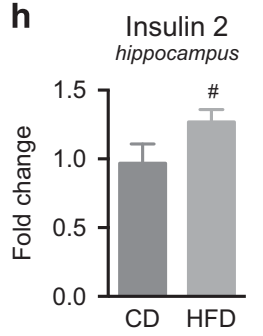

b

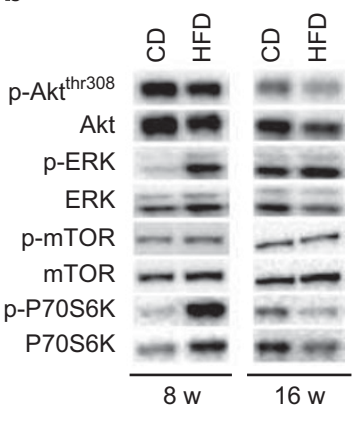

Protein synthesis
cell growth,

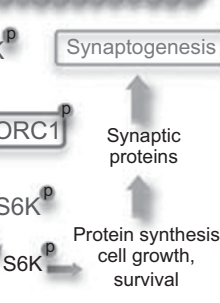

$\mathrm{p}-\mathrm{mTOR}$
hippocampus

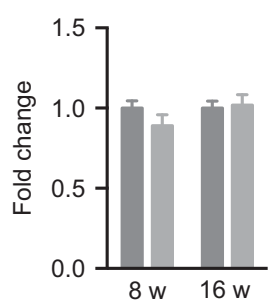

i

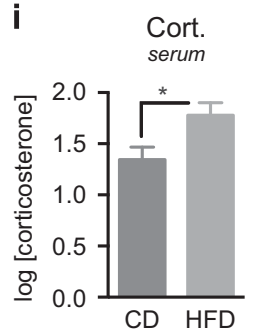

Control Diet

High Fat Diet

f

$\mathrm{p}-\mathrm{P} 70 \mathrm{~S} 6 \mathrm{~K}$
hippocampus

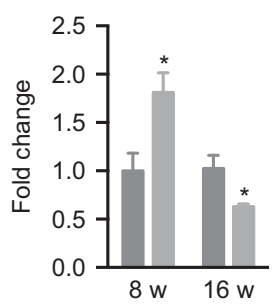

j $\quad \underset{\text { hippocampus }}{\text { CYP11B1 }}$

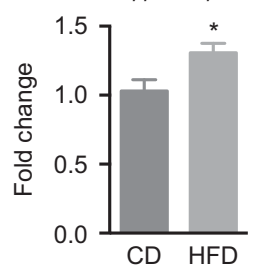

Figure 3 Influence of HFD on insulin, mTORCI signaling, and corticosterone. (a) Schema showing that activation of insulin receptor (IR) activates Phosphatidylinositol 3 kinase (PI3K) pathway, leading to Akt/mTORCI signaling pathway involved in protein synthesis required for long-term synaptic plasticity. Phosphorylation (p) of GSK3 at serine 9 leads to the inhibition of GSK3 activity. Levels of the active form of GSK3 (dephosphorylated) are increased in depression, diabetes, and Alzheimer's disease. (b) Representative immunoblots obtained after 8 and 16 weeks of CD or HFD. (c-f) Quantitative results of phospho (p) protein immunoblots are based on total levels of protein for each particular kinase. HFD differentially alters the phosphorylation of Akt, ERK, and P70S6K in the hippocampus, determined by western blot analysis but no change was detected in levels of $m$ TOR. ( $g$ and $h$ ) Levels of insulin receptor and insulin 2 mRNA in hippocampus were determined by qPCR analysis: HFD ( 16 weeks) significantly increased insulin receptor mRNA and tended to increase insulin 2 mRNA. (i) HFD for 16 weeks also increased serum corticosterone levels (log-transformed data), and (j) hippocampal levels of CYPI IBI mRNA. Results are presented as the mean \pm SEM, $n=8$ per group. ${ }^{*} P<0.05$ compared with the control diet group (two-tailed Student's $t$-tests), ${ }^{\#} P=0.08$. CYPI I BI: cytochrome P450, family II, subfamily B, polypeptide I.

decreased after 16 weeks of HFD $(P=0.0197$, Figure 3f). There were no effects on phospho-mTOR in the hippocampus, and no effect on phospho-Akt308, -ERK, and -P70S6K in the PFC (non-shown).

In addition, we found that 16 weeks of HFD increased not only serum corticosterone (CD: $33.06 \mathrm{ng} / \mathrm{dl}$, HFD: $90.33 \mathrm{ng} /$ $\mathrm{dl}, P=0.0190$ on $\log$-transformed values, Figure $3 \mathrm{i}$ ) but also mRNA levels of CYP11B1, which encodes $11 \beta$-hydroxylase, an enzyme required for the synthesis of corticosterone, in the hippocampus $(P=0.0201$, Figure $3 \mathrm{j})$. There was also a trend for HFD to increase levels of CYP11B1 in the PFC $(P=0.0807$, not shown). These findings are consistent with the possibility that elevated HPA activity and corticosterone synthesis in the brain contribute to the behavioral effects of HFD.

\section{Expression of Pro-Inflammatory Cytokines is Increased by HFD}

We examined the influence of HFD on the expression of pro-inflammatory cytokines, including levels of mRNA and protein by qPCR and Multiplex immuno-assay, respectively. The results show that levels of IL-6 (mRNA and protein, $P=0.0324$ and 0.0049 , respectively), IL- $1 \beta$ (mRNA, $P=0.0095$ ), and TNF $\alpha$ (protein only, $P=0.0309$ ) are significantly increased in the hippocampus of rats fed a HFD for 16 weeks compared with controls (Figure 4a). There was also a trend for increased levels of IL-1 $\beta$ protein $(P=0.0704)$. We also examined the expression of ADAM17, a gene involved in regulating proteolytic release from cellular membranes of signaling proteins, including the cytokines 

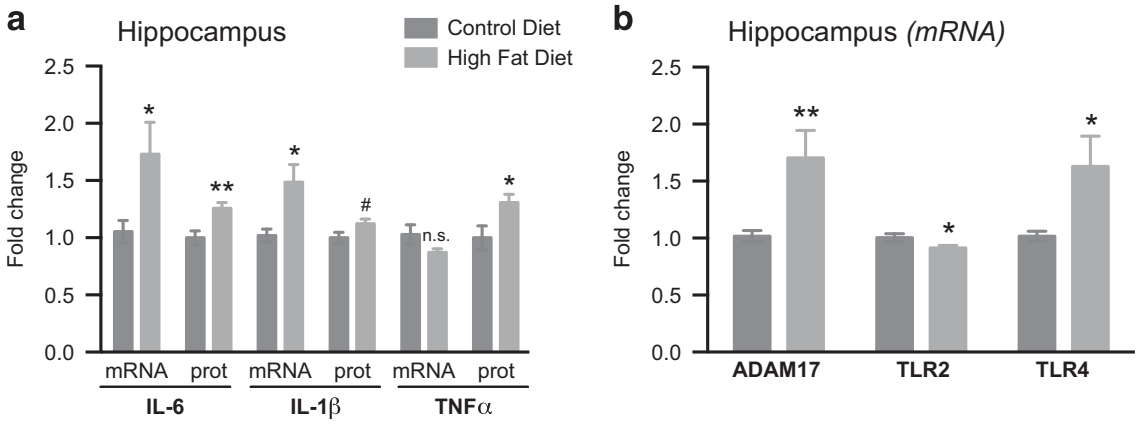

Figure 4 HFD exposure increases inflammatory cytokines and alters TLR expression in the hippocampus. (a) Levels of the inflammatory cytokines IL-6, IL- $\mid \beta$, and TNF $\alpha$ were determined by qPCR (mRNA) and Multiplex assay (proteins). (b) ADAMI 7, TLR2, and TLR4 mRNA levels were also determined by qPCR. Results are presented as the mean \pm SEM, $n=8-16$ per group. $* P<0.05$, $* * P<0.01$, and ${ }^{\#} P=0.07$; NS, nonsignificant compared with the control diet group (two-tailed Student's t-test).

TNF $\alpha$ and IL-6. Levels of ADAM17 mRNA were also increased in the hippocampus of HFD rats $(P=0.0088$, Figure $4 \mathrm{~b}$ ).

Considering that several studies have reported a role for TLR2 and TLR4 in metabolic-related inflammation involving insulin resistance and obesity (Fresno et al, 2011; Könner and Brüning, 2011), we next examined mRNA levels of these two receptors. The results demonstrate a significant increase of TLR4 mRNA $(P=0.0375)$ and a significant decrease of TLR2 mRNA expression in the hippocampus $(P=0.0346$, Figure $4 \mathrm{~b}$ ), with no changes in PFC (not shown).

\section{Ketamine Rapidly Reverses Anxiety and Anhedonic Behavior Caused by HFD and Increases mTORC1 Signaling in the PFC}

A single low dose of the NMDA receptor antagonist ketamine produces rapid antidepressant actions within hours $(2-4 \mathrm{~h})$ that lasts for $\sim 7$ days in treatment-resistant depressed patients (Berman et al, 2000; Krystal et al, 2013; Romeo et al, 2015). Based on these findings, we examined the influence of ketamine $(20 \mathrm{mg} / \mathrm{kg}$, i.p. $)$ in rats exposed to 15-18 weeks of HFD (Figure 5a). Because the behavioral analysis required several weeks of testing, we administered a booster dose of ketamine every 6 days. One day after the first injection, animals were food deprived and tested the day after for anxiety-like behavior in the NSFT. Two-way ANOVA revealed a significant effect of diet $\left(\mathrm{F}_{(1,20)}=7.533\right.$, $P<0.05)$ and drug $\left(\mathrm{F}_{(1,20)}=7.104, P<0.05\right)$. Interestingly, HFD-induced anxiety was reversed by ketamine, which decreased the latency to feed in the center of the open field (HFD-saline: $469.97 \mathrm{~s}$, HFD-ketamine: $305.4 \mathrm{~s}$ ). A post hoc Fisher's LSD test showed that the HFD-saline and HFDketamine groups differed significantly at $P<0.05$ (Figure 5b); there was no significant effect of HFD or ketamine on home cage consumption (Figure $5 \mathrm{c}$ ). In the OFT (performed on day 21), there was a nonsignificant trend toward increased entries in the center in the HFD-ketamine group $\left(\mathrm{F}_{(1,25)}=4.112 ; P=0.053\right.$; HFD-saline: 2.85 , HFD-ketamine: 6.14; Figure $5 \mathrm{~d}$ ). There were no significant effects of HFD or ketamine in the EPM (Figure 5e), consistent with the results presented in Figure 2.

In the FUST (performed on day 4 after ketamine injection), two-way ANOVA revealed a significant effect of
$\operatorname{diet}\left(\mathrm{F}_{(1,27)}=7.956, P<0.01\right)$, with a significant decrease in HFD-saline animals compared with CD-saline (CD-saline: 49.99 s, HFD-saline: $15.51 \mathrm{~s}, P<0.001$, Figure $5 \mathrm{~g}$ ). HFD rats treated with a single dose of ketamine spent more time sniffing (HFD-ketamine: $35.52 \mathrm{~s}, P<0.01$ compared with HFD-saline). In the SPT model of anhedonia, two-way ANOVA for sucrose preference revealed a significant main effect of diet $\left(\mathrm{F}_{(1,17)}=5.566, P<0.05\right)$, but not for drug. HFD reduced the preference for sucrose solution (CD-saline: 89.27\%, HFD-saline: $37.3 \%$ ) and post hoc LSD test showed that $\mathrm{CD}$-saline and HFD-saline differed significantly at $P<0.05$ (Figure 5f). In addition, HFD-induced anhedonia (performed on day 19) was reversed by ketamine (HFDketamine: $72.79 \%, P<0.05$ compared with HFD-saline). Overall, the results indicate that ketamine reverses the anhedonic behaviors resulting from HFD. Since some of the behavioral tests were performed after the booster doses of ketamine (every 6 days of testing), it is possible that the some responses were due to this repeat dosing regimen.

In the NOR, two-way ANOVA for exploration of novel object revealed a significant main effect of diet $\left(\mathrm{F}_{(1,20)}=15.28, P<0.001\right)$, confirming that HFD caused impairments compared with CD saline (CD-saline: $68.35 \%$, HFD-saline: $49.91 \%, P<0.01$, Figure $5 \mathrm{~h}$ ). The results also reveal a main effect of drug $\left(\mathrm{F}_{(1,20)}=12.53, P<0.005\right)$, with ketamine improving the performance of HFD rats (HFDketamine: $66.96 \%, P<0.05$ compared with HFD-saline). Finally, the results show that neither HFD nor ketamine influenced locomotor activity (Figure 5i).

Based on studies demonstrating that the rapid antidepressant actions of ketamine require increased mTORC1 signaling, we analyzed the effects of ketamine on both the PFC and hippocampus of rats fed long-term CD or HFD, using the same rats described for behavioral analysis (ketamine administered week 14, booster doses every 6 days for 31 days; last injection $24 \mathrm{~h}$ before killing the rats). ANOVA results showed an effect of the diet on phosphoERK levels in the hippocampus $\left(\mathrm{F}_{(1,28)}=10.25, P<0.01\right)$, and post hoc LSD test showed that CD groups and HFD-saline differed significantly at $P<0.05$. We also observed a trend toward a decrease of phospho-P70S6K in HFD-saline compared with CD groups, which did not reach significance. Interestingly, decreases of phospho-Akt, phospho-ERK, and phospho-P70S6K were observed in the PFC of HFD-saline 

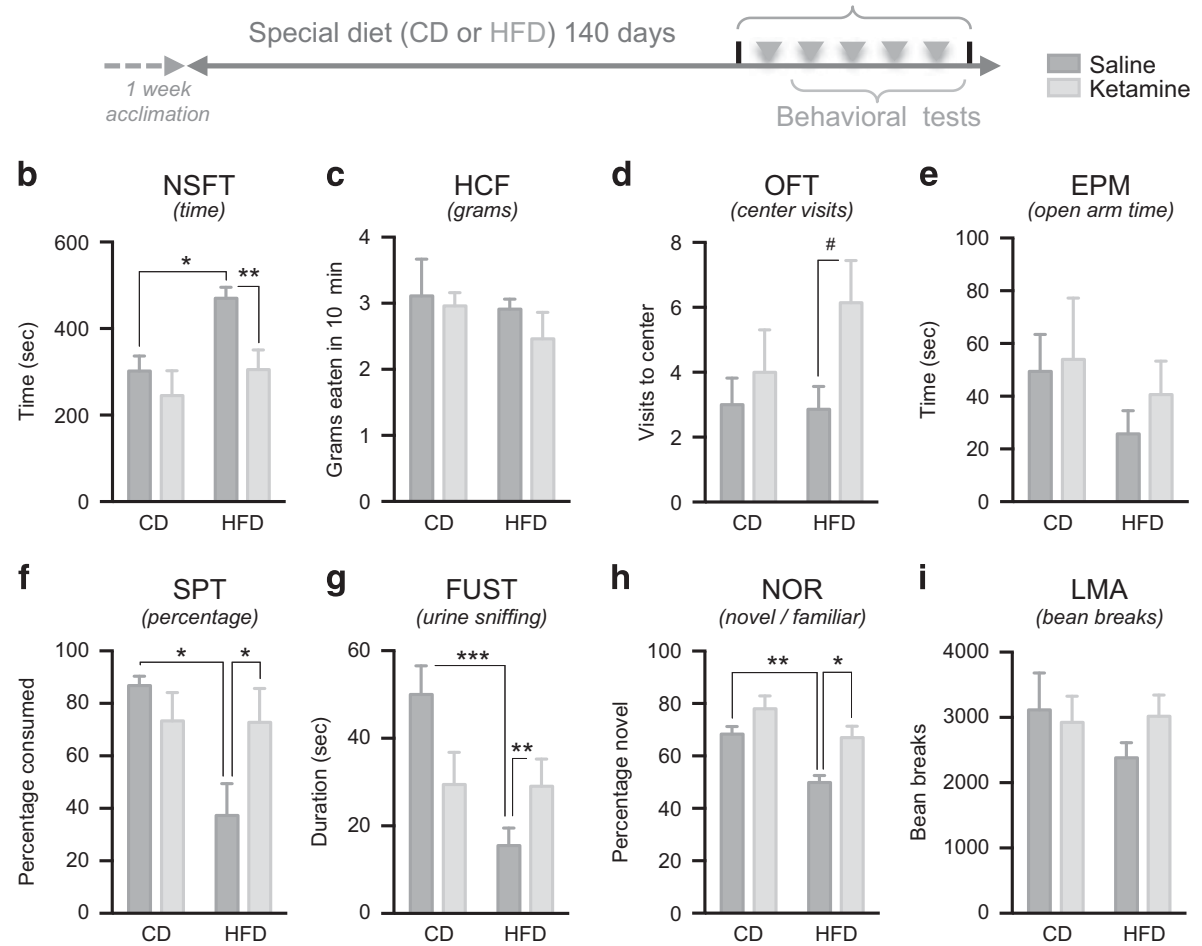

Figure 5 Ketamine administration rapidly reverses anxiety and anhedonia caused by HFD. (a) Experimental paradigm: rats were exposed to CD or HFD for 140 days, and were then administered ketamine $(20 \mathrm{mg} / \mathrm{kg})$ or saline once every 6 days over the last $3 \mathrm{I}$ days of behavioral testing. The influence of HFD and ketamine on anxiety was assessed in the (b) novelty suppressed feeding test (NSFT) and (c) its control home cage feeding (HCF), (d) open field test (OFT) and (e) elevated plus maze (EPM). Anhedonia was examined in the ( $f$ ) sucrose preference test (SPT) and (g) female urine sniffing test (FUST). (h) Novel object recognition (NOR) results. (i) Neither diet nor treatment influenced locomotor activity (LMA). Results are presented as the mean \pm SEM, $n=8$ per group. ${ }^{*} P<0.05$; $* * P<0.01$, **** $P<0.00$ I, ANOVA and Fisher's least significant difference post hoc test for multiple-group comparisons.

rats (Figure 6a-d), and two-way ANOVA showed a significant effect of drug on phospho-Akt $\left(\mathrm{F}_{(1,28)}=6.924\right.$, $P<0.05)$. Post hoc Fisher's LSD test showed that the HFDsaline differed significantly from HFD-ketamine and CDsaline groups at $P<0.05$ (Figure 6e). Levels of phospho-ERK were significantly decreased in the HFD-saline group relative to all other groups $(P<0.05$, Figure 6f); ANOVA revealed a main effect of diet $\left(\mathrm{F}_{(1,28)}=4.333, \mathrm{~F}<0.05\right)$ and an interaction between diet and treatment $\left(\mathrm{F}_{(1,28)}=4.873, P<0.05\right)$. Two-way ANOVA for phospho-P70S6K revealed a significant main effect of diet $\left(\mathrm{F}_{(1,26)}=9.579, P<0.01\right)$ and drug $\left(\mathrm{F}_{(1,26)}=10.70, P<0.001\right)$; post hoc LSD test showed that phospho-P70S6K was significantly increased in CDketamine group relative to all other groups $(P<0.05$, Figure 6h); in addition, there was a trend for a decrease of phospho-P70S6K in the HFD-saline compared with CDsaline $(P=0.071)$, which was nearly reversed by ketamine $(P=0.063)$.

\section{P2X7 Receptor Antagonist Treatment Blocks Anxiety-Like Behavior Caused by HFD and Decreases Inflammation in Hippocampus}

The P2X7 receptor is expressed on innate immune cells in the periphery and brain, including microglia, and serves as a sensor of ATP that activates the inflammasome, stimulating the processing, and release of IL-1 $\beta$ (Iwata et al, 2013). We have recently found that blockade of the P2X7 blocks the behavioral effects of chronic stress on anhedonic behavior (Iwata et al, 2016). Here we tested the influence of a P2X7 receptor antagonist on HFD-induced behaviors. Rats were fed control or HFD for 15 weeks, and were administered vehicle or the P2X7R antagonist A804598 for the last 25 days (Figure 7a). The results show that A804598 administration significantly decreases latency to feed in both the CD and the HFD rats in the NSFT (Figure 7b) while not interfering with HCF (not shown). Two-way ANOVA revealed a significant effect of diet $\left(\mathrm{F}_{(1,20)}=6.099 ; P<0.01\right)$ and drug $\left(\mathrm{F}_{(1,20)}=13.58 ; P<0.001\right)$. We did not observe a significant increase in latency in the HFD group, as was observed in Figure $7 \mathrm{~b}$, possibly due to increased latency in the $\mathrm{CD}$ group that could result from repeated vehicle injection stress.

In the OFT, we found that HFD decreased the number of visits and time in the center, and that A804598 administration reversed these anxiety behaviors (HFD-vehicle: $1 \mathrm{~s}$, HFD-P2X7: $14.14 \mathrm{~s}$, Figure 7d). There was an interaction between diet and drug for these two measures $\left(\mathrm{F}_{(1,24)}=6.664\right.$, $P<0.05$, Figure $7 c$; and $F_{(1,23)}=4.293, P<0.05$, Figure $\left.7 d\right)$. Consistent with these findings, in the EPM, A804598 administration increased the number of visits to the open arms (HFD-vehicle: 1.42, HFD-P2X7: $4.14 \mathrm{~s}$, Figure 7e) and the time spent in open arms (HFD-vehicle: $4.66 \mathrm{~s}$ HFD-P2X7: 42.64 s, Figure 7f). Two-way ANOVA revealed a 
a
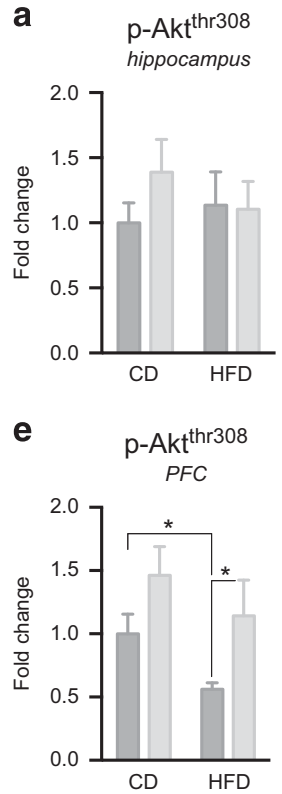

b

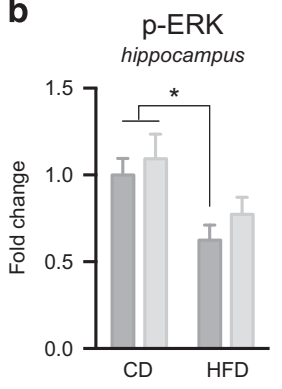

f

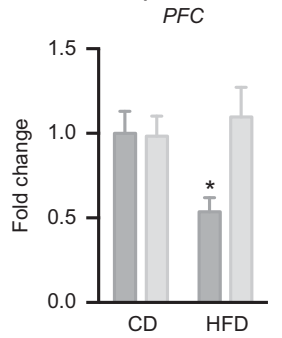

C

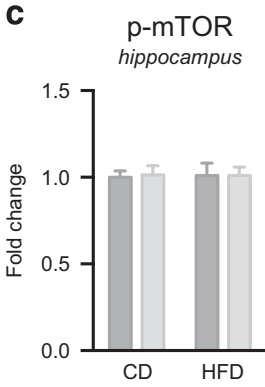

g

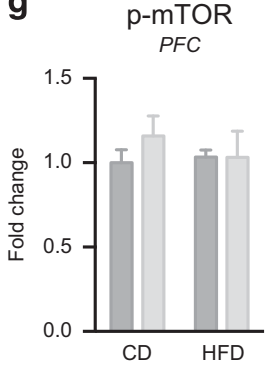

d
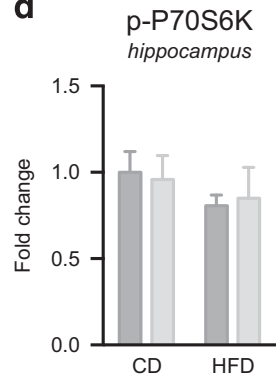

h

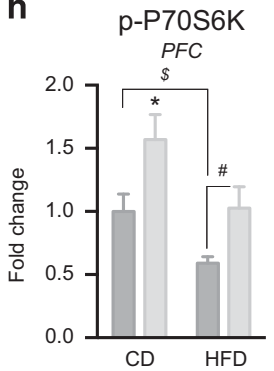

Figure 6 Ketamine treatment activates mTOR signaling in rat prefrontal cortex (PFC). Effects of ketamine (20 mg/kg, i.p. injected 5 times once every 6 days) or saline, on phospho-Akt308, phospho-ERK, phospho-mTOR and phospho-P70S6K in the (a-d) hippocampus and (e-h) prefrontal cortex (PFC) in groups of rats fed CD or HFD for 16 weeks and who underwent behavioral testing before killing the rats ( $c$ Figure 5). Values represent mean \pm SEM ( $n=8$ animals). ${ }^{*} P<0.05 ;{ }^{\$} P=0.07$ I, ${ }^{\#} P=0.063$, ANOVA and Fisher's least significant difference post hoc test for multiple-group comparisons.

significant effect of drug for the number of entries and time spent in open arms $\left(\mathrm{F}_{(1,24)}=4.306, P<0.05\right.$ and $\mathrm{F}_{(1,22)}=$ $7.815, P<0.05$, respectively). There were no effects of A804598 or HFD on locomotor activity (Figure $7 \mathrm{~g}$ ). Apart from the NSFT, there were no effects of A804598 administration in the $\mathrm{CD}$ control group in the other anxiety tests.

\section{DISCUSSION}

The goal of this study was to examine the impact of a HFD on the biological processes that contribute to the development of both T2D and depression- and anxiety-related behaviors. Our results demonstrate that HFD causes behavioral deficits similar to those observed in chronic stress models of depression, including increased anxiety and anhedonia. These behavioral changes are associated with reduced levels of phospho-P70S6K, increased expression of inflammatory cytokines, and disrupted insulin signaling in the hippocampus combined with elevated serum corticosterone and central synthetic capacity. We also report that ketamine rapidly reverses the behavioral deficits that result from long-term exposure to HFD (anxiety-like behavior and anhedonia), and that these effects are associated with activation of phospho-Akt, phospho-ERK, and phosphoP70S6K in the PFC. In addition, chronic administration of a P2X7 receptor antagonist blocks HFD-induced anxiety behaviors.

The HFD male rodent model is the closest clinically relevant model of T2D (Kumar et al, 2012; Panchal and Brown, 2011). We chose a very high content of fat reported to induce systemic hyperglycemia and hyperinsulinemia within 1 week that persists over time (Ahrén and Pacini,
2002; Winzell and Ahrén, 2004). In our study, we confirmed that animals responsive to HFD develop chronic hyperglycemia and impairments of glucose clearance. Hippocampus is one of the structures that has the highest density of insulin receptors and neuronal insulin resistance has been reported showing disruption of insulin-signaling in the hippocampus both in T2D humans and HFD rats (Ghasemi et al, 2012; Pratchayasakul et al, 2011). Here we show that HFD increases insulin receptor expression and produces a trend for increased insulin 2 expression in the hippocampus, effects that could result from a compensatory mechanism to reduced insulin insensitivity.

Although clinical studies and meta-analyses highlight a bi-directional relationship between T2D and depression (Anderson et al, 2001; Champaneri et al, 2010, Golden 2007, Holt et al, 2014; van Dooren et al, 2013), the pathological cross-talk underlying the increased risk of developing these highly comorbid conditions remain unknown, and effective therapeutic agents are still needed. Consistent with previous studies showing elevated HPA axis activity in men and women (Björntorp and Rosmond, 2000; Ghasemi et al, 2012), as well as in rodents (André et al, 2014; Pratchayasakul et al, 2011; Souza et al, 2007), our results show that long-term HFD increases serum corticosterone levels and hippocampal expression of CYP11B1, which encodes the corticosterone synthetic enzyme11-hydroxysteroid dehydrogenase. In line with increased corticosterone levels, we found robust behavioral effects of HFD on anxiety, consistent with previous work in rats and mice demonstrating that a high-energy diet leads to anxiety associated with increased HPA activity (Pratchayasakul et al, 2011). Interestingly, when we assessed anhedonic behavior, we observed that chronic HFD decreased the preference for a pleasurable 


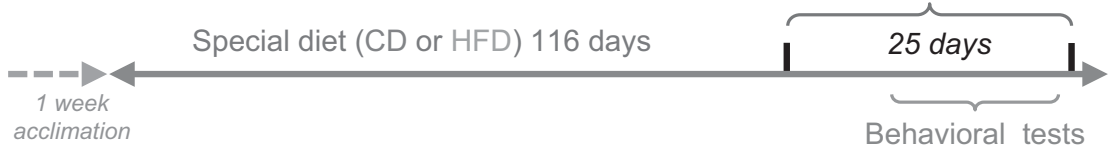

Vehicle P2X7

b

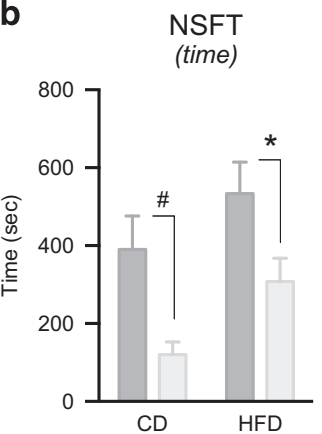

e

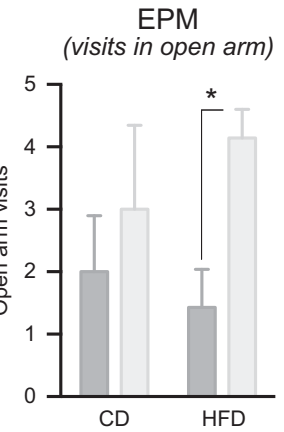

C
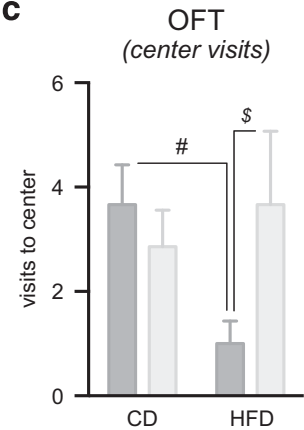

f

f

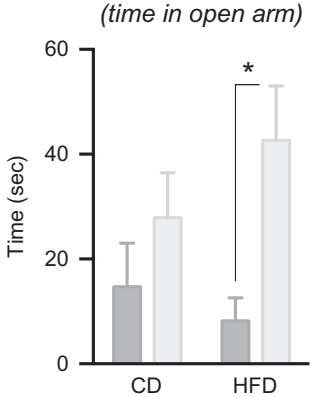

d
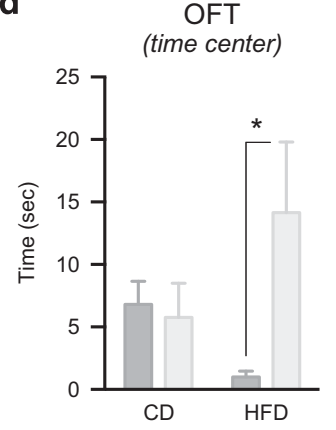

g

LMA (bean breaks)

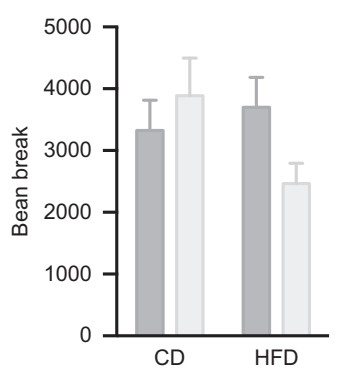

Figure 7 P2X7 receptor antagonist reverses anxiety caused by HFD. (a) Experimental paradigm: rats were exposed to CD or HFD for I I 6 days, and were then administered A-804598 (5 mg/kg, i.p.) or vehicle, twice daily for the last 25 days. Behavioral tests were conducted to assess the effects of HFD and drug treatment on anxiety in the (b) novelty suppressed feeding test (NSFT), (c and d) open field test (OFT), and (e and f) elevated plus maze. ( $g$ ) Neither diet nor treatment influenced locomotor activity (LMA). Results are presented as the mean $\pm S E M, n=8$ per group. ${ }^{*} P<0.05 ;{ }^{*} P=0.06$, ANOVA and Fisher's least significant difference post hoc test for multiple-group comparisons.

sweetened solution. Further, we measured reward-seeking behavior in the FUST, which is associated with activation of brain reward centers, confirming the reward content of this cue (Malkesman et al, 2010). In our study, we found that HFD rats displayed reduced interest toward the reward incentive stimuli (ie female urine), and the lack of difference in the latency to sniff the first time between the groups indicates that the high-fat food did not cause olfactory alterations. These findings are consistent with reports that diet-induced obesity reduces dopamine responses in brain reward areas (Johnson and Kenny, 2010; Stouffer et al, 2015) and extend the results of a previous report in mice (Sharma and Fulton, 2013). The lack of exploration of a novel object in HFD rats during the NOR task could be due to anhedonia (decreased reward of exploring a novel object), although this could also be due to increased anxiety and neophobia. Taken together, the results demonstrate that long-term HFD leads to the development of anxiety and anhedonia, two core symptoms of depression observed in humans and in rodents exposed to chronic stress.
Remarkably, HFD-induced behavioral deficits were rapidly reversed by a single dose of ketamine, as we observed in the NSFT, FUST, and NOR. We further confirmed and extended these results by using different types of tests measuring the same parameters following booster injections of ketamine (every 6 days), confirming in our model the robust antidepressant and anxiolytic action of ketamine. We thus believe that ketamine is a rapid acting agent able to reverse pro-depressant and anxiogenic effects caused by long-term high-fat food consumption.

Next, to elucidate the neurobiological alterations that underlie the anxiety and depressive-like behaviors caused by HFD, we investigated several signaling pathways and systems that play a role in stress and depression. One of these is mTORC1, a serine/threonine protein kinase that integrates signaling from growth factors, energy, and nutrient levels to regulate translation and new protein synthesis (Dwyer et al, 2015; Li et al, 2010; Ota et al, 2014b; Sengupta et al, 2010). Ketamine rapidly stimulates $\mathrm{mTORC} 1$ signaling in the PFC of rats, and this pathway is involved in its rapid synaptic 
and behavioral actions ( $\mathrm{Li}$ et al, 2010, 2011). Conversely, inhibition of mTORC1 causes atrophy of neurons similar to the effects of chronic stress (Ota et al, 2014b). Here we show that HFD causes transient alterations of phospho-Akt, phospho-ERK, and phospho-P70S6K in the hippocampus, with increases in phospho-ERK and phospho-P70S6K at an intermediate time point before the brain becomes insulin resistant (Pratchayasakul et al, 2011). Then, longer HFD exposure (16 weeks), which causes central insulin resistance, reduces phospho-P70S6K signaling. Deficits of phosphoERK and phospho-P70S6K, as well as phospho-Akt were also observed in the PFC, and were reversed by ketamine administration. Increased signaling via these pathways has been linked with increased synaptic connectivity in the PFC and reversal of synaptic deficits caused by chronic stress (Li et al, 2010), and could represent novel therapeutic targets for illnesses associated with HFD and T2D.

Among the different cascades of signaling pathways activated directly or indirectly by insulin, TLRs could also serve as a bridge in the relationship between obesity, insulin resistance, and depression. TLRs are danger-associated molecular pattern receptors that also interact with insulin, inflammation, and mTORC1 signaling pathways (Ghasemi et al, 2012). These receptors have a number of functions, including regulation of the inflammasome and expression of inflammatory cytokines, protein synthesis, cell survival, and apoptosis (Hanke and Kielian, 2011). Here we show that TLR2 mRNA is decreased in hippocampal tissue of HFD animals. In line with this finding, a study reported that null mutation of TLR2 causes metabolic syndrome even when mice are on a low-fat diet (Ehses et al, 2010; Kuo et al, 2011), suggesting a link between TLR2 and insulin signaling. In contrast, TLR4 expression in obese mice and obese and diabetic patients negatively correlates with insulin sensitivity (Könner and Brüning, 2011), and in the present study, we found that hippocampal TLR4 expression increased under chronic HFD exposure. Overall, these findings provide compelling support that long-term HFD impairs not only peripheral, but also brain insulin signaling/glucose homeostasis and synaptic plasticity. TLR2/4 could contribute to the anxiety and depressive-like phenotypes in our animals.

Current theories suggest that depression, often triggered by stress, is associated with increased inflammatory response (Müller, 2014; Wager-Smith and Markou, 2011). Increased levels of inflammatory cytokines have been extensively reported in depressed patients and rodent models of chronic stress and associated with the severity of depression (Dowlati et al, 2010; Dunn and Swiergiel, 2005; Howren et al, 2009; Iwata et al, 2013). Moreover, inflammation is one of the main characteristics of obesity and insulin resistance, not only in the blood and peripheral systems, but also in the central nervous system (De Souza et al, 2005; Erion et al, 2014; Posey et al, 2009) and pro-inflammatory cytokines interact with multiple pathways known to influence mood regulation and the development of depression (Haroon et al, 2012). Here we found that HFD significantly increased the expression of pro-inflammatory cytokines (IL-6, IL-1 $\beta$, $\operatorname{TNF} \alpha$ ). We also find that HFD increased the expression of ADAM17, which is involved in the proteolic release of these cytokines (Menghini et al, 2013) and is reported to be increased in post-mortem brain tissue of patients with psychiatric disorder (Marballi et al, 2012).
Recently, we found that stress increases extracellular ATP, leading to the activation of $\mathrm{P} 2 \mathrm{X} 7$ receptors and release of inflammatory cytokines via the innate immune complex Nucleotide-binding domain, leucine-rich repeat, pyrin domain containing 3 (NLRP3) inflammasome (Iwata et al, 2013). The NLRP3 inflammasome can act as a sensor for a broad range of danger-related molecules. Interestingly, NLRP3 knock-out mice are resistant to the development of HFD-induced obesity (Vandanmagsar et al, 2011), suggesting that NLRP3 activation through danger signals contributes to inflammation and insulin resistance. Based on this evidence, we tested whether blockade of innate immune activation could alter the adverse side effects of HFD using a P2X7 receptor antagonist. The results show that chronic administration of a P2X7 receptor antagonist significantly decreases anxiety of HFD rats, confirming the anxiolytic properties of drugs targeting inflammation in our model. Further experiments are needed to assess the effects of P2X7 antagonist treatment on anhedonic-behavior on HFD rats. Together, these findings indicate that blockade of P2X7 and inflammasome activation represents a viable therapeutic strategy for anxiety associated with T2D and HFD. The results also highlight a key pathological mechanism that can serve as a bridge for depression and diet-induced metabolic disorders and should help clinicians to better understand psychiatric complications in these populations.

\section{CONCLUSIONS}

The present study highlights new findings on risk factors that serve as a bridge between T2D and psychiatric illness. More precisely, the results provide new neurobiological evidence to support aspects of the neuroinflammatory hypothesis of depression based on HFD-induced anxiety and depressive behavior, and offers novel insights about potential treatments. In this study, we report that HFD exposure alters energy homeostasis and insulin/mTORC1 signaling pathways that are known to regulate synaptic plasticity, TLR expression, and pro-inflammatory cytokine production. Targeting these pathways, including the mTORC1 signaling cascade (ie, ketamine) as well as the innate immune system (ie, P2X7 antagonist) reverse the behavioral deficits caused by HFD. These findings identify novel treatment strategies for patients with metabolic disorder or T2D and who are more susceptible to anxiety and depression. The results thereby open new avenues to understand the links between nutrition, insulin dysfunction, inflammation, and psychiatric behavioral disorders.

\section{FUNDING AND DISCLOSURE}

This work was supported by the Connecticut Mental Health Center, Yale University, and by Eli Lilly and Company through the Lilly Research Award Program (LRAP). P2X7 antagonist A804598 was provided by Eli Lilly, Indianapolis, IN. The authors declare no conflict of interest.

\section{ACKNOWLEDGMENTS}

We thank Eunyoung Bang for assistance in the preparation of P2X7 antagonist. 


\section{AUTHOR CONTRIBUTIONS}

S.D. prepared the original draft of the manuscript and was involved in all aspects of the experimental design and research, including execution and analysis of all behavioral, biochemical and molecular experiments. K.T.O. and E.S.W. assisted with sample preparation. K.R. reviewed and helped with manuscript preparation. R.S.D. was involved in all aspects of study design, interpretation of results and preparation of the manuscript and figures.

\section{REFERENCES}

Ahrén B, Pacini G (2002). Insufficient islet compensation to insulin resistance vs. reduced glucose effectiveness in glucose-intolerant mice. Am J Physiol Endocrinol Metab 283: E738-E744.

Anderson RJ, Freedland KE, Clouse RE, Lustman PJ (2001). The prevalence of comorbid depression in adults with diabetes: a meta-analysis. Diabetes Care 24: 1069-1078.

André C, Dinel A-L, Ferreira G, Layé S, Castanon N (2014). Diet-induced obesity progressively alters cognition, anxiety-like behavior and lipopolysaccharide-induced depressive-like behavior: focus on brain indoleamine 2,3-dioxygenase activation. Brain Behav Immun 41: 10-21.

Berman RM, Cappiello A, Anand A, Oren DA, Heninger GR, Charney DS et al (2000). Antidepressant effects of ketamine in depressed patients. Biol Psychiatry 47: 351-354.

Björntorp P, Rosmond R (2000). Obesity and cortisol. Nutr Burbank Los Angel Cty Calif 16: 924-936.

Champaneri S, Wand GS, Malhotra SS, Casagrande SS, Golden SH (2010). Biological basis of depression in adults with diabetes. Curr Diab Rep 10: 396-405.

De Souza CT, Araujo EP, Bordin S, Ashimine R, Zollner RL, Boschero AC et al (2005). Consumption of a fat-rich diet activates a proinflammatory response and induces insulin resistance in the hypothalamus. Endocrinology 146: 4192-4199.

den Heijer T, Vermeer SE, van Dijk EJ, Prins ND, Koudstaal PJ, Hofman A et al (2003). Type 2 diabetes and atrophy of medial temporal lobe structures on brain MRI. Diabetologia 46: 1604-1610.

Dowlati Y, Herrmann N, Swardfager W, Liu H, Sham L, Reim EK et al (2010). A meta-analysis of cytokines in major depression. Biol Psychiatry 67: 446-457.

Duman RS, Li N, Liu R-J, Duric V, Aghajanian G (2012). Signaling pathways underlying the rapid antidepressant actions of ketamine. Neuropharmacology 62: 35-41.

Dunn AJ, Swiergiel AH (2005). Effects of interleukin-1 and endotoxin in the forced swim and tail suspension tests in mice. Pharmacol Biochem Behav 81: 688-693.

Dwyer JM, Maldonado-Avilés JG, Lepack AE, DiLeone RJ, Duman RS (2015). Ribosomal protein S6 kinase 1 signaling in prefrontal cortex controls depressive behavior. Proc Natl Acad Sci USA 112: 6188-6193.

Ehses JA, Meier DT, Wueest S, Rytka J, Boller S, Wielinga PY et al (2010). Toll-like receptor 2-deficient mice are protected from insulin resistance and beta cell dysfunction induced by a highfat diet. Diabetologia 53: 1795-1806.

Erion JR, Wosiski-Kuhn M, Dey A, Hao S, Davis CL, Pollock NK et al (2014). Obesity elicits interleukin 1-mediated deficits in hippocampal synaptic plasticity. J Neurosci Off J Soc Neurosci 34: 2618-2631.

Fresno M, Alvarez R, Cuesta N (2011). Toll-like receptors, inflammation, metabolism and obesity. Arch Physiol Biochem 117: 151-164.

Ghasemi R, Haeri A, Dargahi L, Mohamed Z, Ahmadiani A (2012). Insulin in the brain: sources, localization and functions. Mol Neurobiol 47: 145-171.
Golden SH (2007). A review of the evidence for a neuroendocrine link between stress, depression and diabetes mellitus. Curr Diabetes Rev 3: 252-259.

Hanke ML, Kielian T (2011). Toll-like receptors in health and disease in the brain: mechanisms and therapeutic potential. Clin Sci Lond Engl 1979 121: 367-387.

Haroon E, Raison CL, Miller AH (2012). Psychoneuroimmunology meets neuropsychopharmacology: translational implications of the impact of inflammation on behavior. Neuropsychopharmacol Off Publ Am Coll Neuropsychopharmacol 37: 137-162.

Holt RIG, de Groot M, Golden SH (2014). Diabetes and depression. Curr Diab Rep 14: 491.

Howren MB, Lamkin DM, Suls J (2009). Associations of depression with C-reactive protein, IL-1, and IL-6: a meta-analysis. Psychosom Med 71: 171-186.

Iwata M, Ota KT, Duman RS (2013). The inflammasome: pathways linking psychological stress, depression, and systemic illnesses. Brain Behav Immun 31: 105-114.

Iwata M, Ota KT, Li XYi, Sakaue F, Li N, Dutheil S et al (2016). Psychological stress activates the inflammasome via release of ATP and stimulation of the P2X7 receptor. Biol Psychiatry (in press).

Jacka FN, Cherbuin N, Anstey KJ, Sachdev P, Butterworth P (2015). Western diet is associated with a smaller hippocampus: a longitudinal investigation. BMC Med 13: 215.

Johnson PM, Kenny PJ (2010). Dopamine D2 receptors in addiction-like reward dysfunction and compulsive eating in obese rats. Nat Neurosci 13: 635-641.

Kamiyama K, Wada A, Sugihara M, Kurioka S, Hayashi K, Hayashi $\mathrm{T}$ et al (2010). Potential hippocampal region atrophy in diabetes mellitus type 2: a voxel-based morphometry VSRAD study. Jpn J Radiol 28: 266-272.

Könner AC, Brüning JC (2011). Toll-like receptors: linking inflammation to metabolism. Trends Endocrinol Metab TEM 22: 16-23.

Koo JW, Russo SJ, Ferguson D, Nestler EJ, Duman RS (2010). Nuclear factor-kappaB is a critical mediator of stress-impaired neurogenesis and depressive behavior. Proc Natl Acad Sci U S A 107: 2669-2674.

Krystal JH, Sanacora G, Duman RS (2013). Rapid-acting glutamatergic antidepressants: the path to ketamine and beyond. Biol Psychiatry 73: 1133-1141.

Kumar S, Singh R, Vasudeva N, Sharma S (2012). Acute and chronic animal models for the evaluation of anti-diabetic agents. Cardiovasc Diabetol 11: 9.

Kuo L-H, Tsai P-J, Jiang M-J, Chuang Y-L, Yu L, Lai K-TA et al (2011). Toll-like receptor 2 deficiency improves insulin sensitivity and hepatic insulin signalling in the mouse. Diabetologia 54: 168-179.

Last D, Alsop DC, Abduljalil AM, Marquis RP, de Bazelaire C, $\mathrm{Hu} \mathrm{K}$ et al (2007). Global and regional effects of type 2 diabetes on brain tissue volumes and cerebral vasoreactivity. Diabetes Care 30: 1193-1199.

Lephart ED, Setchell KDR, Handa RJ, Lund TD (2004). Behavioral effects of endocrine-disrupting substances: phytoestrogens. ILAR J 45: 443-454.

Leys C, Ley C, Klein O, Bernard P, Licata L (2013). Detecting outliers: Do not use standard deviation around the mean, use absolute deviation around the median. J Exp Soc Psychol 49: 764-766.

Li N, Lee B, Liu R-J, Banasr M, Dwyer JM, Iwata M et al (2010). mTOR-dependent synapse formation underlies the rapid antidepressant effects of NMDA antagonists. Science 329: 959-964.

Li N, Liu R-J, Dwyer JM, Banasr M, Lee B, Son H et al (2011). Glutamate NMDA receptor antagonists rapidly reverse behavioral and synaptic deficits caused by chronic stress exposure. Biol Psychiatry 69: 754-761.

Macbeth AH, Edds JS, Young WS (2009). Housing conditions and stimulus females: a robust social discrimination task for studying male rodent social recognition. Nat Protoc 4: 1574-1581. 
Machida M, Fujimaki S, Hidaka R, Asashima M, Kuwabara T (2012). The insulin regulatory network in adult hippocampus and pancreatic endocrine system. Stem Cells Int 2012: 959737.

Malkesman O, Scattoni ML, Paredes D, Tragon T, Pearson B, Shaltiel G et al (2010). The female urine sniffing test: a novel approach for assessing reward-seeking behavior in rodents. Biol Psychiatry 67: 864-871.

Marballi K, Cruz D, Thompson P, Walss-Bass C (2012). Differential neuregulin 1 cleavage in the prefrontal cortex and hippocampus in schizophrenia and bipolar disorder: preliminary findings. PLoS One 7: e36431.

Menghini R, Fiorentino L, Casagrande V, Lauro R, Federici M (2013). The role of ADAM17 in metabolic inflammation. Atherosclerosis 228: 12-17.

Müller N (2014). Immunology of major depression. Neuroimmunomodulation 21: 123-130.

Ota KT, Liu R-J, Voleti B, Maldonado-Aviles JG, Duric V, Iwata M et al (2014a). REDD1 is essential for stress-induced synaptic loss and depressive behavior. Nat Med 20: 531-535.

Ota KT, Liu R-J, Voleti B, Maldonado-Aviles JG, Duric V, Iwata M et al (2014b). REDD1 is essential for stress-induced synaptic loss and depressive behavior. Nat Med 20: 531-535.

Panchal SK, Brown L (2011). Rodent models for metabolic syndrome research. J Biomed Biotechnol 2011: 351982.

Posey KA, Clegg DJ, Printz RL, Byun J, Morton GJ, Vivekanandan-Giri A et al (2009). Hypothalamic proinflammatory lipid accumulation, inflammation, and insulin resistance in rats fed a high-fat diet. Am J Physiol Endocrinol Metab 296: E1003-E1012.

Pratchayasakul W, Kerdphoo S, Petsophonsakul P, Pongchaidecha A, Chattipakorn N, Chattipakorn SC (2011). Effects of high-fat diet on insulin receptor function in rat hippocampus and the level of neuronal corticosterone. Life Sci 88: 619-627.

Romeo B, Choucha W, Fossati P, Rotge J-Y (2015). Meta-analysis of short- and mid-term efficacy of ketamine in unipolar and bipolar depression. Psychiatry Res 230: 682-688.

Salim S, Chugh G, Asghar M (2012). Inflammation in anxiety. Adv Protein Chem Struct Biol 88: 1-25.

Schmidt HD, Shelton RC, Duman RS (2011). Functional biomarkers of depression: diagnosis, treatment, and pathophysiology. Neuropsychopharmacol Off Publ Am Coll Neuropsychopharmacol 36: 2375-2394.
Sengupta S, Peterson TR, Sabatini DM (2010). Regulation of the mTOR complex 1 pathway by nutrients, growth factors, and stress. Mol Cell 40: 310-322.

Sharma S, Fulton S (2013). Diet-induced obesity promotes depressive-like behaviour that is associated with neural adaptations in brain reward circuitry. Int J Obes 200537 382-389.

Souza CG, Moreira JD, Siqueira IR, Pereira AG, Rieger DK, Souza DO et al (2007). Highly palatable diet consumption increases protein oxidation in rat frontal cortex and anxiety-like behavior. Life Sci 81: 198-203.

Stienstra R, van Diepen JA, Tack CJ, Zaki MH, van de Veerdonk FL, Perera D et al (2011). Inflammasome is a central player in the induction of obesity and insulin resistance. Proc Natl Acad Sci U S A 108: 15324-15329.

Stouffer MA, Woods CA, Patel JC, Lee CR, Witkovsky P, Bao L et al (2015). Insulin enhances striatal dopamine release by activating cholinergic interneurons and thereby signals reward. Nat Commun 6: 8543.

van Dooren FEP, Nefs G, Schram MT, Verhey FRJ, Denollet J, Pouwer F (2013). Depression and risk of mortality in people with diabetes mellitus: a systematic review and meta-analysis. PLoS One 8: e57058.

Vandanmagsar B, Youm Y-H, Ravussin A, Galgani JE, Stadler K, Mynatt RL et al (2011). The NLRP3 inflammasome instigates obesity-induced inflammation and insulin resistance. Nat Med 17: 179-188.

Wager-Smith K, Markou A (2011). Depression: a repair response to stress-induced neuronal microdamage that can grade into a chronic neuroinflammatory condition? Neurosci Biobehav Rev 35: 742-764.

Warden CH, Fisler JS (2008). Comparisons of diets used in animal models of high-fat feeding. Cell Metab 7: 277.

Warner-Schmidt JL, Duman RS (2007). VEGF is an essential mediator of the neurogenic and behavioral actions of antidepressants. Proc Natl Acad Sci U S A 104: 4647-4652.

Winzell MS, Ahrén B (2004). The high-fat diet-fed mouse: a model for studying mechanisms and treatment of impaired glucose tolerance and type 2 diabetes. Diabetes 53 (Suppl 3): S215-S219.

Zarate CAJ, Singh JB, Carlson PJ, Brutsche NE, Ameli R, Luckenbaugh DA et al (2006). A randomized trial of an $\mathrm{N}$-methyl-D-aspartate antagonist in treatment-resistant major depression. Arch Gen Psychiatry 63: 856-864. 